\title{
Buprenorphine as an adjuvant to local anesthetics in peripheral nerve blocks
}

\author{
Omar Viswanath ${ }^{1,2,3}$ and Ivan Urits ${ }^{4}$ \\ ${ }^{1}$ Valley Anesthesiology and Pain Consultants, Phoenix, AZ, USA \\ ${ }^{2}$ Department of Anesthesiology, University of Arizona College of Medicine-Phoenix, Phoenix, AZ, USA \\ ${ }^{3}$ Department of Anesthesiology, Creighton University School of Medicine, Omaha, NE, USA \\ ${ }^{4}$ Department of Anesthesia, Critical Care, and Pain Medicine, Beth Israel Deaconess Medical Center, Harvard Medical School, \\ Boston, MA, USA
}

\section{LETTER TO THE EDITOR}

We read Dr. Jeon's article, "The use of adjuvants to local anesthetics: benefit and risk" [1], with great interest. "Therefore, perineural injection of adjuvants combined with local anesthetics has evolved for prolongation of the local anesthetic effect, without exhibiting the adverse effects common to opioids, epinephrine, dexmedetomidine, or steroids." The article goes on to explain the adverse effects of each type of adjuvant when administered perineurally: "For example, the use of opioids such as fentanyl or morphine increases the risk of respiratory depression, nausea and vomiting, and pruritus."

We wanted to take this opportunity to mention another adjuvant that can be used in peripheral nerve blocks that falls under the opioid category, but differs in that it is not a pure opioid agonist - buprenorphine. Buprenorphine is a semisynthetic opioid that is approximately thirty times more potent than morphine, acting as a partial agonist at the mu receptor and as an antagonist at the kappa receptor.
A 2017 meta-analysis by Schnabel et al. [2] consisting of 13 randomized control trials, totaling 685 patients, tested the efficacy and safety of buprenorphine in peripheral nerve blocks. The study concluded that the addition of buprenorphine to a local anesthetic peripheral nerve block prolonged postoperative analgesia for approximately 8 hours but also significantly increased the risk for postoperative nausea and vomiting (PONV).

In our personal experience, buprenorphine as an adjuvant has yielded positive results with prolonged duration of blocks with no evidence of PONV as a result in our patients. It is important to note the lack of PONV may simply be due to a small sample size of patients with peripheral nerve blocks with buprenorphine added to it. Buprenorphine as an adjuvant to local anesthetic peripheral nerve blocks is another option for clinicians to utilize, but the benefits of a prolonged duration of a block and subsequent analgesia must be weighed against the possibility of the manifestation of its most common adverse effects, namely PONV.

Received December 14, 2018. Revised January 4, 2019. Accepted January 9, 2019.

Correspondence to: Omar Viswanath

Valley Anesthesiology and Pain Consultants, 645 E. Missouri Avenue, Suite 300, Phoenix, AZ 85012, USA

Tel: +1-630-881-9591, Fax: +1-602-648-4361, E-mail: viswanoy@gmail.com

ORCID: https://orcid.org/0000-0002-6124-7037

(a) This is an open-access article distributed under the terms of the Creative Commons Attribution Non-Commercial License (http:// creativecommons.org/licenses/by-nc/4.0/), which permits unrestricted non-commercial use, distribution, and reproduction in any medium, provided the original work is properly cited.

(C) The Korean Pain Society, 2019 


\section{CONFLICT OF INTEREST}

No potential conflict of interest relevant to this article was reported.

\section{ORCID}

Omar Viswanath, https://orcid.org/0000-0002-6124-7037 Ivan Urits, https://orcid.org/0000-0002-3652-6085

\section{REFERENCES}

1. Jeon $Y H$. The use of adjuvants to local anesthetics: benefit and risk. Korean J Pain 2018; 31: 233-4.

2. Schnabel A, Reichl SU, Zahn PK, Pogatzki-Zahn EM, MeyerFrießem $\mathrm{CH}$. Efficacy and safety of buprenorphine in peripheral nerve blocks: a meta-analysis of randomised controlled trials. Eur J Anaesthesiol 2017; 34: 576-86. 